\title{
Riesgo y vulnerabilidad en Llano Largo, Acapulco: la tormenta Henriette
}

\section{Risk and vulnerability in Llano Largo, Acapulco: Hurricane Henriette}

\author{
América Rodríguez-Herrera \\ Manuel RuZ-VARgas \\ Berenise Hernández-Rodríguez*
}

\begin{abstract}
This article discusses the social experience generated from risk, out of the devastation caused by Tropical Storm Henriette in 2007, in the various settlements and housing developments and other sectors of Llano Largo and surrounding area located in the iconic Zona Diamante (Diamond Zone), of Acapulco city. It addresses disaster management by identifying the actors involved, affected population, construction companies and the state. Despite the social and cultural heterogeneity, villagers have noticed emerging organizational dynamics though incipient, contribute to identify the way to understate and place themselves in a reality of risks. The state, with a policy focused on the emergency, fails to integrate the processes of reconstruction that is held by private organizations, whose interests lie in taking advantage of the resulting vulnerability, while the state only serves the population in poverty with focused help.
\end{abstract}

Keywords: risk, vulnerability, floods, social actors.

\section{Resumen}

En este artículo se aborda la experiencia social generada frente al riesgo, a partir de los estragos provocados por la tormenta Henriette en el ańo 2007, en Llano Largo y otros sectores aledańos localizados en la emblemática Zona Diamante de la ciudad de Acapulco. Se identificó a los actores involucrados en las gestiones tras el desastre: población afectada, las empresas constructoras y el Estado. A pesar de la heterogeneidad social y cultural, los pobladores han realizado dinámicas organizativas que aunque incipientes, aportan para identificar el camino que les ayude a comprender y ubicarse en una realidad plagada de riesgos. El Estado, con una política centrada en la emergencia, no logra integrar los procesos de reconstrucción, lo que queda en manos de organismos privados cuyo interés no es la superación de la vulnerabilidad resultante. Únicamente atiende a la población en condiciones de pobreza con ayuda focalizada. Mientras los riesgos son para los pobres, las empresas continúan siendo las ganadoras en este reparto de ganancias.

Palabras clave: riesgo, vulnerabilidad, inundaciones, actores sociales.

* Universidad Autónoma de Guerrero, México. Correos-e: america_rodríguez_herrera@hotmail. com; comaru_2002@yahoo.com.mx; silvia_berenise@hotmail.com. 


\section{El riesgo como construcción social}

Se ha señalado que el riesgo es una de las características fundamentales de las sociedades contemporáneas, donde éste se impone como un ordenador de la vida social. La sociedad de riesgo se produce por las transformaciones ocurridas en el mundo con el avance de la globalización y la revolución tecnológica (Beck, 2006), en ella el miedo es una preocupación compartida por toda la población sin distinción de clases, esto se debe a que los avances tecnológicos ocurren con una velocidad exponencial, mientras que las respuestas de los grupos humanos en cuanto a la percepción de los riesgos no se desarrollan en la misma proporción. Como señala Gaspar Mairal-Buil, "estamos rodeados de riesgos con una dimensión tan extraordinaria que rebasa con creces al conjunto de la experiencia humana" (1999: 2).

Concebido así, el riesgo se plantea como una construcción social, generada por los grupos humanos, por tanto, la generación de riesgos depende de una diversidad de factores: sociales, políticos, económicos, tecnológicos o de poder, relacionados a menudo con la presencia de amenazas naturales que conducen a una situación de sufrimiento. En este sentido los riesgos se ven en una perspectiva amplia y compleja, que trasciende la valoración fisicalista (Hewit, 1983) que privilegia la observación del impacto de las fuerzas naturales en las colectividades.

Acerca del riesgo existen dos enfoques teóricos que aunque coinciden en que los desastres son socialmente construidos, difieren en el énfasis que colocan ya sea en aspectos estructurales o culturales, y que según García Acosta (2005), en tanto avanzan en ámbitos distintos ofrecen aportes teóricos y metodológicos complementarios que contribuyen a una apreciación integral de los desastres.

Para los primeros la vulnerabilidad es un concepto clave para entender el riesgo y los desastres, ésta resulta de los procesos de desarrollo mal llevados o incompletos, frecuentes en las sociedades latinoamericanas (Wilches, 1983; Maskrey, 1983; Lavell y Franco, 1996), los cuales agregan o predisponen a las colectividades -física y socialmente- a la angustia o a la pérdida, de manera que la confluencia de los riesgos y las amenazas se convierten en desastres (Lavell, 2002). Para estos autores el concepto de desastre es fundamental, ya que de ello se deriva el quehacer frente a un evento extraordinario. En este sentido, la respuesta ante los desastres privilegia soluciones técnicas o se enfoca a la atención de problemas estructurales.

De allí que el Estado como promotor del desarrollo es un actor fundamental en la generación de riesgos y vulnerabilidades, pues el cambio es consustancial al desarrollo; sin embargo, parece que este argumento no se considera en las políticas, ya que en ellas priva la idea de que tanto 
riesgo como vulnerabilidad son daños colaterales del desarrollo (Lavell, 2000), que por un lado conduce a políticas de atención que privilegian el restablecimiento de condiciones normales, lo que incluye también la ayuda focalizada a grupos en situación de pobreza. De acuerdo con esta perspectiva es imposible -como recomiendan Lavell y Franco (1996)_ visualizar los desastres como ventanas al desarrollo, es decir, que de los procesos de gestión se derive un trabajo que se proyecte en opciones integrales que incluyan una perspectiva de desarrollo, en particular porque el componente de participación es sumamente limitado en el esquema gubernamental, de manera que sólo las comunidades con una propuesta organizativa alternativa podrían plantearse procesos de gestión del riesgo que conlleven a un desarrollo local.

Sin embargo la respuesta de la población frente a los desastres no es automática ni lineal; a menudo se ve matizada por las experiencias colectivas, las actitudes, los valores, y en general por los ámbitos normativos, cognoscitivos y simbólicos que cobijan a una sociedad. Pues un territorio se construye también desde la cultura de los grupos sociales establecidos en ese espacio geográfico, donde cada grupo construye sus relaciones y su cotidianeidad a partir de lo que considera riesgoso o seguro.

Para Mary Douglas el riesgo es una construcción cultural de las sociedades producida en su devenir histórico, a partir de la asociación entre las amenazas y determinadas condiciones de vulnerabilidad que se construyen y reconstruyen con el tiempo (Douglas, en García, 2005). Así, la percepción pública del riesgo y su aceptación son construcciones colectivas que incluyen una dimensión histórico-temporal y hacen referencia a una matriz cultural determinada. Evans (1994) añade la importancia de apreciar la temporalidad en la manera en que se procesa el riesgo, lo que nos lleva a las ideas de futuro y la forma como se percibe el cambio, las cuales se combinan para crear un mapa cognitivo que filtra la percepción de riesgo. Por otro lado, llama la atención en la influencia de las pautas culturales occidentales y cómo éstas dan prioridad a la percepción del corto plazo, todo lo cual nos explicaría el por qué de la corta memoria sobre los eventos desastrosos y de sufrimiento colectivo, así como la insistencia de algunos grupos a aferrarse a escenarios de riesgos.

De esta manera, la percepción del riesgo es una construcción colectiva producto de la interacción de los grupos humanos entre sí y con su medio, lo que implica adaptaciones e incluso transformaciones del medio ambiente, sobre la base de conocer su propio territorio. Por ello algunos autores lo relacionan con los procesos de construcción de identidades (Ríos y Múrgida, 2004).

Lo anterior nos muestra la complejidad de relaciones en que se despliegan los actores sociales, donde sus constructos sociales, poderes, jerarquías 
y cotidianeidades intervienen en escenarios de riesgo o desastre. De la misma manera que los proyectos de desarrollo no se enfrentan a espacios vacíos, los afectados por riesgos tampoco parten de cero, sus protagonistas, con base en sus experiencias colectivas, pueden conducir sus espacios de gestión por dinámicas fecundas e insospechadas.

Este artículo aborda la experiencia social generada frente al riesgo, a partir de los estragos provocados por la tormenta Henriette, que asoló Llano Largo y otros sectores aledańos localizados en la emblemática Zona Diamante de la ciudad de Acapulco, los días 31 de agosto al 2 de septiembre de 2007, un espacio de la periferia urbana marcado por la heterogeneidad social y cultural, que ha observado dinámicas de participación que aportaron al impulso de una organización que, aunque incipiente y aun limitada, ha descubierto el camino para comprender y ubicarse en una realidad plagada de riesgos. Pretendemos abordar la participación de los actores fundamentales en los procesos de gestión de la emergencia y la reconstrucción de cara a ese desastre, con la finalidad de identificar los procesos socioculturales que pueden contribuir a generar espacios favorables a la participación y construcción de una percepción que contribuya a enfrentar situaciones de riesgo. Examinar las experiencias locales ayuda a visualizar caminos construidos en la cotidianeidad que pueden contribuir a entender y repensar tantos escenarios de riesgo que en la actualidad afligen a grandes sectores de población en nuestro país y el mundo.

\section{Metodología}

Se aplicó metodología cuantitativa y cualitativa, con la cual se estableció como zona de estudio un polígono delimitado a partir de las características físicas y geográficas y su relación con el entorno social. También tomamos en cuenta la funcionalidad de los espacios, de acuerdo con el plan de desarrollo urbano de Zona Diamante y la georreferenciación a través del uso de sistemas de información geográfico con GPS y la sobreposición en ortofotos del inegi, trabajado en el Arc View 3.2. Para la caracterización de la población se utilizaron datos del INEGI y estadísticas generadas en otros proyectos realizados en la zona. El método cualitativo permitió recabar testimonios sobre historia del lugar así como la percepción de riesgo y gestión del territorio y de los riesgos entre los habitantes. Algunos testimonios se obtuvieron de informantes clave: autoridades, líderes, pobladores y funcionarios, pero también se consultó a población que se escogió de manera aleatoria. 


\section{Acapulco, Llano Largo y el riesgo de inundación}

Acapulco es una ciudad localizada al sur de la República Mexicana, que creció y se posicionó en el mercado de bienes y servicios con el influjo de la actividad turística que se inició durante las primeras décadas del siglo $\mathrm{xx}$. Como otras ciudades intermedias, tuvo un importante incremento demográfico a partir de la década de los años cincuenta del siglo pasado, población atraída por la actividad económica desarrollada en este importante puerto.

El crecimiento poblacional y el desarrollo urbano de esta ciudad encontró un territorio limitado para su expansión, un accidentado terreno pedregoso caracterizado por la articulación de tierras altas y costeras; las primeras marcadas por una de las estribaciones de la Sierra Madre del Sur, que en el conjunto de la ciudad figuran una especie de anfiteatro con pendientes de hasta $45^{\circ}$, en cuya base se encuentra el Valle de la Sabana, y una estrecha franja costera que termina en la bahía de Santa Lucía o de Acapulco, o bien en el mar. Por ello la ciudad se encuentra atravesada por una entretejida red de ríos y barrancas, donde a partir de la década de los cincuenta la mancha urbana ha avanzado sistemáticamente, mediante la invasión, canalización o desvío de ríos, reduciéndolos a cauces pluviales. ${ }^{1}$ Para el año 2007, Juárez (2009), con base en datos de Protección Civil Municipal, dio cuenta de la presencia de 78 cauces en $116 \mathrm{~km}$ correspondientes a la zona urbana y suburbana de la ciudad de Acapulco. En este crecimiento urbano encontramos asentamientos irregulares, establecidos en zonas federales, en las márgenes o bien en los propios cauces.

Tan sólo entre 2004 y 2006, la tasa de crecimiento de la población localizada en estos espacios fue de $445.3 \%$, lo que muestra un preocupante escenario de riesgos en esta ciudad, particularmente porque se encuentra

\section{Cuadro 1}

Viviendas, población y tasa de crecimiento de población en asentamientos irregulares en Acapulco durante el periodo 20042006

\begin{tabular}{ccccc}
\hline Año & Viviendas & Población & $\begin{array}{c}\text { Tasa de } \\
\text { crecimiento } \\
\text { anual }\end{array}$ & $\begin{array}{c}\text { Tasa de } \\
\text { crecimiento } \\
\text { delperiodo }\end{array}$ \\
\hline 2004 & 506 & 2,530 & & \\
2005 & 1,814 & 9,070 & $258.5 \%$ & \\
2006 & 2,759 & 13,795 & $52.09 \%$ & $445.3 \%$ \\
\hline
\end{tabular}

Fuente: Elaboración propia con base en Juárez (2009).

${ }^{1}$ Ríos como el Camarón, Aguas Blancas, Garita. 
en el llamado Cinturón de Fuego del Océano Pacífico; frente a las costas del puerto se localiza la Fosa de Acapulco, donde se juntan la Placa Continental con la Ártica (Placa de Cocos), pero además es una zona ciclogénica que amenaza estacionalmente con fenómenos hidrometeorológicos extraordinarios, de manera que los riesgos son múltiples y diversos: terremotos, tsunamis, deslaves, rocas inestables e inundaciones.

Dentro de la ciudad de Acapulco, nuestra zona de estudio, Llano Largo, se ubica en Zona Diamante, al sureste de la ciudad, en una estrecha franja costera próxima al lecho marino, la cual es receptora de las principales corrientes que bajan de las partes altas, del parque El Veladero que soporta una precipitación pluvial de 1,314 mm (CNA estación 12-001), y del río La Sabana que atraviesa todo el municipio de Acapulco para desembocar en la laguna de Tres Palos, que constituye un sistema lagunar junto con la laguna Negra de Puerto Marqués, a la cual se conecta por un canal meándrico (figura I).

En la fisiografía de Llano Largo confluyen humedales, lagunetas y meandros que contribuyen al desalojo de las aguas pluviales. Forma parte de un ecosistema estuarino, un importante espacio productivo de agricultura de humedad y pesca.

De acuerdo con el proyecto de desarrollo de Zona Diamante, se impulsaría una exclusiva zona que permitiría reposicionar la oferta turística de Acapulco como un centro de primer orden (Ruiz, 1989). Se construyó el boulevard de Las Naciones que constituyó el eje vial que uniría este

\section{Figura I}

\section{Zona de estudio: Llano Largo, Unidad Luis Donaldo Colosio, fraccionamientos aledańos y boulevard de Las Naciones}

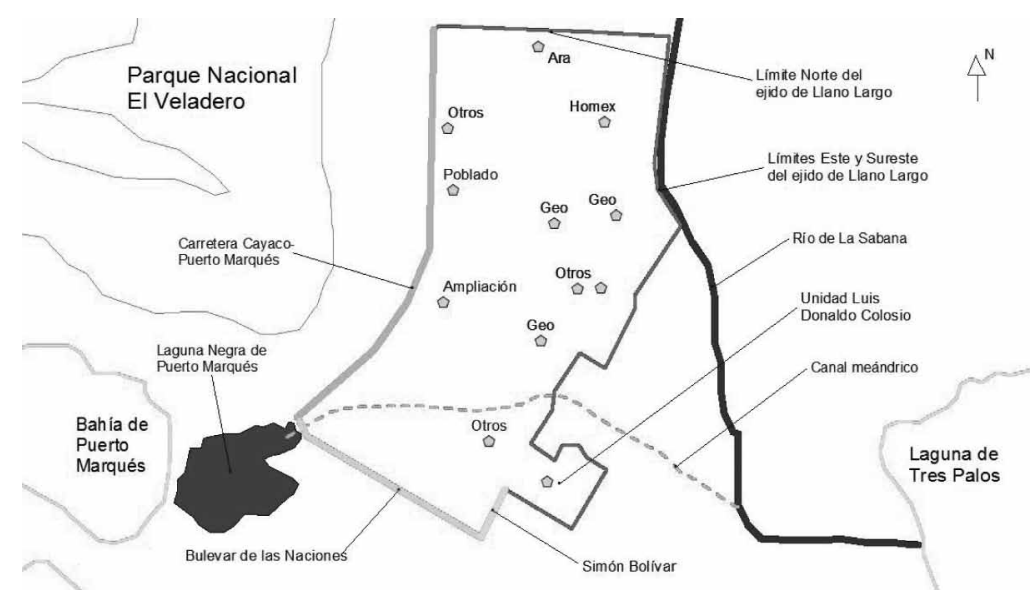

Fuente: Elaboración propia con datos de campo georreferenciados. 
sector con la ciudad de Acapulco y el aeropuerto internacional de esta ciudad; también se definieron áreas de servicios turísticos y para uso habitacional en los antiguos espacios ejidales y expropiados. Este último fue el destino del ejido de Llano Largo y otros terrenos de propiedad privada aledaños, que terminaron integrándose a la mancha urbana.

Los primeros desarrollos habitacionales construidos fueron la Unidad Luis Donaldo Colosio, Villas El Paraíso I, II y III, residencial Los Arcos y Joyas del Marqués sobre terrenos expropiados al ejido de Puerto Marqués, durante el gobierno del presidente Miguel Alemán Valdés (1946-1952) (Jacinto et al., 2009: 50). Las tierras ejidales de Llano Largo fueron liberadas al mercado de tierras urbano mediante el Proceso de Certificación de Derechos Ejidales y Solares (Procede) que sobrevino a las modificaciones al artículo 27 constitucional de 1992, el cual se completó a finales de los noventa; para preparar la construcción se rellenaron humedales y se canalizaron barrancas, pero la mayor transformación se realizó en el antiguo ejido de Llano Largo, a partir del año 2000. En sólo siete años se vendió $70 \%$ de las tierras y, según una nota periodística, se había completado la construcción de 10,600 viviendas (La Jornada Guerrero, 10 de octubre de 2007), el resultado fue la modificación no sólo de la topografía, sino también del sistema natural de desalojo de las aguas pluviales que escurrían hacia este espacio. De esta manera, era cosa de tiempo para que las lluvias extraordinarias generaran inundaciones que dañaran los bienes de la creciente población establecida en el antiguo territorio ejidal.

En cuanto a la dotación de infraestructura se observa que los primeros asentamientos construidos cuentan con servicios conectados a la red pública, incluidas plantas tratadoras de aguas residuales; mientras que los fraccionamientos construidos en Llano Largo no cuentan con este servicio, cada empresa ha perforado sus propios pozos profundos para suministrar el agua de uso doméstico. En cuanto a las aguas residuales, éstas se conectan a la red pública que conduce a la planta de tratamiento de Miramar, que dejó de funcionar en 2005. Así, las aguas residuales conectan directamente con los humedales localizados en Ampliación Llano Largo, próximos al canal meándrico, lo que genera problemas sanitarios y ambientales. En tanto la población usuaria de servicios en este sector se incrementa, no sólo vía habitaciones sino también establecimientos comerciales o de servicio, la cantidad de aguas residuales tienden a aumentar y a estancarse, amenazando con contaminar pozos artesianos utilizados por la población originaria de Llano Largo, de manera que estos cambios también han tenido consecuencias ambientales en el territorio

En el ámbito cultural se observa una transformación en la composición demográfica y social, con el desplazamiento de población originaria y el ingreso de nuevos habitantes al lugar que incluso se concretó al interior 
del antiguo asentamiento de Llano Largo, en la medida que aun los predios para vivienda entraron al mercado urbano. La desarticulación del ejido pronto dejó a los pobladores originarios sin sus tradicionales organizaciones, lo que afectó las relaciones entre los antiguos pobladores, que además vieron mermadas sus relaciones comunales.

Asimismo, el ingreso de población foránea en los fraccionamientos propició una dinámica social de segmentación y polarización entre los nuevos y los antiguos pobladores. A pesar de que en principio el plan contemplaba la construcción de una zona habitacional para la población local, el resultado fue diferente: las casas construidas pasaron a satisfacer la demanda de segundas viviendas. Éstas se ofrecían en unidades amuralladas con albercas y jardines colectivos, lo que imprime un contraste con las condiciones de vida de la población originaria, además de la presencia de formas de vida diversas que no encuentran en el antiguo territorio puntos de articulación o identificación. Por otro lado, muchos de los nuevos pobladores sólo visitan sus casas durante las temporadas vacacionales o fines de semana, lo que influye en el entorno y en las dinámicas organizativas.

El conteo del INEGi de 2005 muestra que en el polígono estudiado predomina población establecida que cuenta con empleo en el sector formal (52.45\%), mientras que el sector más populoso se localiza en el primer grupo de desarrollos habitacionales construidos, es decir, la Unidad Colosio y aledaños con 16,074 habitantes, mientras que el sector de Llano Largo contaba en ese entonces con 3,556 habitantes. Si se tratara de caracterizar las condiciones económicas de los habitantes, los datos de una encuesta socioeconómica realizada a finales de 2009 en el espacio estudiado (Rodríguez et al., 2010) mostraron que predominan los grupos de población que reciben menos de dos salarios mínimos. Esto nos muestra, por un lado, la poca presencia de pobladores de segunda vivienda establecidos en el lugar y, por otro, el entorno de pobreza y polarización que rodea estos enclaves habitacionales. Durante la tormenta Henriette ocurrida del 31 de agosto al 2 de septiembre de 2007, a la vulnerabilidad física y social de nuestra zona de estudio se agregó la vulnerabilidad generada en el contexto mayor: el desbordamiento del río La Sabana y la barranca El Colacho, lo que provocó que el agua entrara con fuerza e inundara gran parte del espacio contenido en nuestra zona de estudio.

\section{La gestión del desastre: actores y escenarios}

\subsection{La población}

Las inundaciones tomaron por sorpresa a la mayoría de la población establecida en Llano Largo y zonas aledañas; los antiguos pobladores ubi- 
cados en el antiguo asentamiento de Llano Largo no esperaban que el agua se introdujera en sus casas, aunque la zona siempre ha sido inundable; esto es porque el pueblo se estableció en los puntos relativamente altos y dejaron los bajos para el uso agrícola. Por otro lado, la presencia de los humedales contribuía al desalojo de las aguas durante las lluvias extraordinarias. En la memoria colectiva de la antigua población están presentes los huracanes Cosme (1989) y Paulina (1997), cuando la población fue evacuada en lanchas. Narran experiencias traumáticas de Paulina, como la presencia de cadáveres de animales y personas arrastradas desde los puntos altos por el río La Sabana; en ambos casos el evento ocurría en todo el territorio de Acapulco. Situación diferente fue la tormenta Henriette de 2007, cuyo desastre -aunque presente en otros municipios del Estado- se localizaba en el caso de la ciudad de Acapulco en determinados puntos de la Zona Diamante, donde ellos habían tenido las mayores afectaciones. Esto proporcionó a los pobladores de este espacio una significación especial, el reconocimiento de la vulnerabilidad específica que ahora caracteriza su territorio.

Según testimonios obtenidos, cuando durante el meteoro los antiguos pobladores se dieron cuenta que el agua entraba por ciertos lugares, hicieron conciencia de que la antigua percepción de los riesgos era inadecuada; Pablo, un residente, nos dijo: "Cuando el agua entró por lugares inesperados, allí nos dimos cuenta de que el Llano ya no era igual”. Ellos consideran que los cambios en el espacio y sus efectos han sido provocados por las constructoras, pero reconocen que el caos también se presenta en el ámbito mayor: el agua les entró por la barranca El Colacho, es decir, ahora reciben el agua que proviene de otros puntos del Valle de la Sabana, pues la solución más fácil para los constructores fue desviar las corrientes hasta este cauce. Durante la inundación los vecinos pusieron costales de arena, pero su esfuerzo fue insuficiente. En el antiguo poblado las inundaciones generaron daños, sobre todo en las construcciones cuyos materiales o estructuras eran deficientes. Los más afectados fueron los habitantes de las partes más bajas dentro y fuera del poblado, en el sector conocido como Ampliación Llano Largo y La Alborada de Llano Largo, el primero atravesado por humedales y el canal meándrico que une las lagunas de Tres Palos y Negra de Puerto Marqués. Algunos puntos de estos lugares permanecieron anegados cerca de un mes.

Los nuevos pobladores se enfrentaron a diversas experiencias, los que habitan en el sector de la Unidad Colosio y Villas El Paraíso I, II y III fueron de los más expuestos al desbordamiento del río La Sabana, dado que este sector es populoso y debido a su escasa altitud ( 2 metros snm) los drenajes son superficiales, de manera que durante la inundación el agua pluvial se mezcló con las aguas residuales. Según comenta Xóchitl, una 
informante, la calle Simón Bolívar (camino de Llano Largo a la Colosio) se transformó en un cauce por el que se desplazaba libremente el agua: "Semejaba un embudo, donde el agua arrastraba carros, muebles, animales muertos, objetos diversos, y pets", que eran un peligro para las personas que con el agua a la cintura trataban de desplazarse por las calles. Los fraccionamientos de Geo y Homex también fueron inundados por las aguas del río La Sabana y la barranca El Colacho. Cabe mencionar que los habitantes de estos fraccionamientos comentan que antes de la inundación se sentían muy seguros en sus casas, pues las empresas les aseguraron que habían tomado precauciones acerca de posibles inundaciones: la construcción de bardas perimetrales, así como que sus casas estaban edificadas sobre un piso levantado, les daba más seguridad que al resto previamente construido. Esta afirmación se vino abajo cuando el primer fraccionamiento que se inundó fue Costa Dorada (Homex), el agua se estancó al llegar a las bardas de Geo que separan ambos fraccionamientos, éstas actuaban como represas pero luego que se rompieron: el agua se adueñó del territorio Geo. Fue entonces cuando muchos habitantes de estos fraccionamientos se dieron cuenta de la gravedad de la situación, pues el agua traía peces, camarones y fauna asociada al río. En ese momento trataron sin éxito de salir hacia un lugar seguro, algunos quedaron entrampados en sus casas, desorientados, pues las vialidades eran cauces.

Tanto los antiguos como los nuevos pobladores ignoraban el tamaño del riesgo de inundación, no obstante todos, pero en particular los nuevos pobladores, conforme pasaron las horas pudieron concretar iniciativas y organizarse para preservar la integridad de las personas. Debido a que los fraccionamientos están más bajo la influencia del río La Sabana y el arroyo El Colacho, las inundaciones les afectaron con mayor severidad, de allí que en la madrugada del 1 de septiembre, en el fraccionamiento Costa Dorada los vecinos se organizaron para evacuar a las personas, a quienes ubicaron en una casa de dos pisos donde tenían más protección, se organizaron comidas y despensas comunes, vigilancia por turnos y ayuda a los heridos o enfermos. Lo mismo ocurrió en otros fraccionamientos localizados en el sector este del polígono. Xóchitl, una informante, comentó que entonces surgieron muchos liderazgos y se reforzaron los vínculos vecinales: "Dicen que si los buenos momentos unen, los malos unen más, yo cuando me refiero a mi vecina, me refiero a una persona que está muy al pendiente, que comparte... fue una experiencia muy intensa”.

Brigadas conformadas por Protección Civil Municipal y del estado, la Secretaría de Salud y Unidades de la Marina se presentaron para evacuar a la población el día 1 de septiembre y ubicaron a las personas en albergues establecidos en la escuela primaria Luis Donaldo Colosio y en la iglesia de Puerto Marqués. En el caso de los afectados de los fraccionamientos, 
fueron llevados por las empresas a hoteles como el Aca Bay, Fiesta Inn y Copacabana, donde permanecieron durante una semana. La evacuación se realizó en lanchas de la Marina y con maquinaria pesada de las empresas Geo y Homex. Durante la evacuación no se tomaron en cuenta las experiencias organizativas de la población, y una vez instalados en sus refugios, todo el tiempo fueron asistidos y desmovilizados.

Las brigadas de Protección Civil incorporaron a la población afectada, a las empresas constructoras y a las ubicadas en el boulevard de Las Naciones en las tareas de desalojo de las aguas; se abrieron canales (Ampliación Llano Largo) y las barras de El Revolcadero en Puerto Marqués y Barra Vieja de la Laguna de Tres Palos, aun así, buena parte de la zona de estudio estuvo inundada durante una semana, mientras que en los puntos más bajos esta situación permaneció a lo largo de un mes. Aparentemente, los espacios de participación que ofrece Protección Civil se coloca en tareas puntuales, al cabo de las cuales las personas se desmovilizan, aspecto que ha sido analizado por Rodríguez (2003). El componente de seguridad nacional que enmarcas las políticas de cara a la gestión de los desastres se relaciona más en la emergencia que en la reconstrucción o mitigación de los efectos del desastre. Fue posible observar esto en el caso de la gestión de las inundaciones provocadas por Henriette, donde el papel de los organismos públicos fue muy patente durante la emergencia, mientras que en la reconstrucción asumieron un perfil bajo, su relación con la población se tornó conflictiva y contradictoria, mientras surgían otros organismos de la esfera privada.

El regreso de los afectados a su casa fue por diversas vías, en el caso de los fraccionamientos, la empresa Geo trasladó a sus colonos del hotel donde permanecieron refugiados, no sin antes mostrarles las brigadas que trabajaban en la restauración de los jardines, cableado, pintura y de las vialidades. Una de las afectadas interpretó esto como una respuesta que pretendía acallar las demandas de la población, sobre todo porque la empresa estaban en plena construcción de nuevos fraccionamientos; por otro lado, como las casas fueron adquiridas mediante créditos, tenían la posibilidad de apelar al pago de los seguros. Al regreso los residentes pudieron apreciar el monto de sus pérdidas, tras lo cual se iniciaron las protestas y la organización de los afectados. Para los antiguos pobladores, tanto el regreso como la superación de sus daños y pérdidas corrieron por su propia cuenta. El ayuntamiento, la Secretaría de Desarrollo Social (Sedesol) o los políticos eran los únicos a quienes podían recurrir, y con los cuales, como veremos, desarrollaron relaciones conflictivas.

Los nuevos pobladores se articularon en torno a sus organizaciones de condóminos, que en sus inicios pretendían administrar los espacios comunes de los fraccionamientos: jardines, albercas, vigilancia, limpieza. 
En un principio los representantes realizaron gestiones ante las oficinas públicas, pues se sentían defraudados por las empresas, al haberles proporcionado viviendas en zonas de riesgo; también frente a las constructoras y el Infonavit o el Fovissste, instancias que les proporcionaron los créditos, con la finalidad de presionarlas para que contribuyeran al pago de los seguros.

Fue un periodo de intensa movilización de la población, donde surgieron y se fortalecieron liderazgos. En este lapso llegaron los líderes políticos de los partidos Revolucionario Institucional (PRI) y de la Revolución Democrática (PRD), que ofrecían ayuda y aprovechaban para atender y ampliar clientelas. Pero muchos de los nuevos pobladores son originarios de las ciudades del centro de México, con un capital social y cultural que tiene percepciones y experiencias de riesgo y desastre, así como de ejercicio de la ciudadanía diferente a la de los locales. Con niveles económicos y de escolaridad e información más altos, así como relaciones políticas que les permitían tener acceso a funcionarios públicos o privados. Entre los antiguos pobladores también se presentó un trabajo conjunto entre las personas establecidas en los fraccionamientos y otros propietarios que se desenvolvían en la ciudad de México, lo que les facilitaba hacer trámites colectivos. Las protestas, plantones y encerronas de funcionarios fueron contra el ayuntamiento y las constructoras, primero para que los reubicaran en una zona segura, posteriormente solicitaban la devolución de su dinero y la cancelación de sus créditos.

Sin embargo, algunos trámites tuvieron que hacerse en forma individual y ante distintas instituciones, ya que los créditos les habían sido otorgados por aseguradoras diferentes, lo que no contribuyó al fortalecimiento de sus organizaciones; de manera que la dinámica organizativa tuvo sus altas y bajas. Tampoco hubo un acercamiento entre las organizaciones de condóminos de los distintos fraccionamientos y menos aún con los antiguos pobladores; sin embargo, la propuesta de organización, los objetivos y las perspectivas de trabajo conjunto estaban puestas. De hecho, en el verano de 2009 se tuvo la oportunidad de asistir a una reunión de condóminos, donde el punto central era la discusión de medidas de prevención, entre las que se encontraba el diseño de una alerta temprana, rutas de evacuación y medidas de presión para lograr la construcción de las vialidades. Nuevas inundaciones presentadas en junio de 2009 durante la tormenta Andrés, que afectó en forma particular al fraccionamiento Misión del Mar de la constructora Ara, también generaron una intensa movilización de los condóminos y lucha contra la empresa. En el proceso identificaron que además de haber construido sobre cauces, la empresa había incumplido con la dotación de una infraestructura social básica, la presión llegó a tal punto que tuvo que intervenir el ayuntamiento como garante de los acuerdos entre condóminos y la empresa. 
Así, tenemos que entre los nuevos pobladores las organizaciones de condóminos se proyectan como grupos involucrados en la gestión para prevenir el riesgo, aunque todavía en forma limitada, centradas en la problemática propia. Caso contrario son los nuevos pobladores que permanecen inmovilizados, los pocos líderes toman como ámbitos de lucha el campo que ofrecen los políticos y los medios de comunicación, que como ellos mismos reconocen, "casi nunca ponen en los noticieros lo que ellos denuncian" (Polo). En este reparto de riesgos, como señala Beck (2006), le correspondía la mayor parte a los que menos tienen, mientras que las ganancias se concentraban en las empresas que movilizaban el capital. El Estado, por su parte, con sus políticas focalizadas trataba de combatir los dańos colaterales del desarrollo (Lavell, 2000).

De esta manera la vida de los antiguos y nuevos pobladores quedó definida por el Estado y las empresas constructoras, quienes han marcado los cambios en el territorio. Siguiendo el objetivo de la valorización de capitales, la ganancia ha sido el motor principal de la construcción y de la definición de un nuevo uso del espacio. Para Rodríguez (2003), en la práctica pública "el territorio en los planes es acotado como un factor de localización, físico y espacial [...] no se analiza la construcción del territorio y las modalidades de ocupación, apropiación y uso del suelo ni el efecto de las políticas económicas, urbanas y sociales que inciden en la configuración de mayores riesgos e incremento de la vulnerabilidad y sus efectos territoriales en regiones y ciudades. Es decir, imaginar el riesgo como un fenómeno multifactorial no es el fuerte de la agenda pública, con razón Mairal-Buil (1999) señala que como las comunidades carecen de poder, el desarrollo termina siendo decidido por el Estado y los grupos económicos poderosos; la ausencia de mecanismos de participación en las decisiones que afectan a la población de un territorio, contribuyen a generar dinámicas socioculturales -como en Llano Largo- marcadas por la segmentación y polarización social que en nada contribuye a generar espacios de identidad y organización para enfrentar la realidad de múltiples escenarios de riesgos.

\subsection{Las empresas, las aseguradoras y los políticos}

Las constructoras que operan en Llano Largo son empresas subsidiarias de las corporaciones inmobiliarias ligadas al capital nacional e internacional; cubren el mercado de viviendas de interés social y medio, por lo que frecuentemente trabajan con Infonavit y Fovissste. Se consideran "empresas socialmente responsables en cuanto al cuidado del medio ambiente y de las comunidades donde trabajan" (ver portales de internet Geo y Homex). Geo es la inmobiliaria que inauguró la construcción de viviendas en la zona de 
estudio, es la responsable de los primeros desarrollos habitacionales y la que propuso el concepto de segundas viviendas en la zona, que se generalizó con la entrada de empresas como Homex, Ara y otras menores a partir del año 2000 en las tierras recién adquiridas en Llano Largo.

Desde el principio las empresas entraron en procesos de competencia y especulación no sólo para adquirir las mejores ubicaciones y cantidades de terrenos, sino también la clientela. Todas ponían énfasis en la calidad de las viviendas que construían, sin poner mucha atención en el entorno (entrevistas con ingenieros responsables de obras), o en el efecto que tuviera su plan constructor. Durante la emergencia y la superación de ésta, las empresas participaron de manera activa junto a las brigadas organizadas por Protección Civil en la evacuación de sus propios afectados, en el desalojo de las aguas y en el retorno de la población a sus viviendas, sin embargo, su participación en los procesos de negociación para la reconstrucción se llevó a cabo en medio de tensión y conflicto con los habitantes.

Cabe señalar que las empresas enfrentaban la presión política desde los órganos del Estado: el Congreso de la Unión (LX, año II, primer periodo ordinario) y el Congreso del estado (El Sur, 13 de noviembre). También enfrentaban presiones de tipo económico, para el 2007 las empresas no habían completado sus planes de construcción en la zona, por lo que la inundación les resultaba un gran inconveniente en sus proyectos de comercialización.

En el caso de Geo los directivos fueron muy prudentes, invirtieron en la rehabilitación de los espacios comunes y en la infraestructura de servicios. Frente a las demandas de la población, nunca dijeron no, aunque no todo lo cumplieron. En el caso de Homex los conflictos fueron mayores, en parte porque la matriz de esta empresa se encuentra en Culiacán y no siempre las directrices llegaron oportunamente. Esto dio lugar a desencuentros con los afectados que llegaron a tomar las instalaciones y encerrar a sus funcionarios para que resolvieran su situación. Esto marcó las relaciones, de manera que, según testimonios de algunos funcionarios de esta empresa, nunca decían que no y tenían que hacer rifas de enseres domésticos, DVD, computadoras, etc., para contener nuevas protestas o acciones de los condóminos. Según un funcionario de Homex, la empresa terminó el año con saldo rojo debido a la inversión que realizó en la restauración del fraccionamiento, por lo que se empeñaron en una agresiva campaña de ventas en ciudades del centro de México, lo que les permitió recuperarse en el transcurso de 2008.

El trabajo de reconstrucción se desarrollo con la participación de las compañías aseguradoras en los fraccionamientos. Para ello las instituciones crediticias enviaron a sus peritos para que realizaran una evaluación de los daños, lo cual era indispensable para hacer efectivos los seguros. 
Los primeros en llegar fueron los de Infonavit. Una representante de esta institución afirma que se atendieron las demandas de los afectados que tenían sus créditos con la institución, a través del seguro Atlas, en un total de 700 casas (licenciada Irma Arias, Departamento Jurídico). Aparentemente el Fovissste tuvo menos presencia. Sin embargo, algunas notas periodísticas muestran que la respuesta de las aseguradoras también se obtuvo por la presión que hizo la población: plantones en el ayuntamiento, las empresas y en las mismas aseguradoras (La Jornada, 4 de octubre de 2007).

Por su parte, los políticos aparecieron en la escena desde los primeros días que los habitantes retornaron a sus viviendas, les ofrecieron abrir puertas y atraer recursos para la reconstrucción del lugar. Los lugares donde más se hicieron presentes fue en los fraccionamientos y en el populoso sector de la Unidad Colosio. Políticos como Manuel Añore Baños se presentó con su fundación Juntos para Mejorar Acapulco para asistir a los afectados, así como representantes del PRD, lo que generó divisiones entre los condóminos. Un funcionario de Homex comenta que hubo contactos entre los políticos y las empresas, que proporcionaron dinero para que fueran repartidas despensas entre los habitantes, con el propósito de contener a la población en los momentos de mayor movilización en contra de estas últimas.

\subsection{El Estado}

Uno de los efectos del huracán Paulina de 1997 fue sensibilizar acerca de la necesidad de contar con un plan de desarrollo urbano efectivo para la ciudad, por ello, con la concurrencia de los gobiernos federal y estatal se integró un Comité de Desarrollo Urbano destinado a actualizar el Plan Director de Desarrollo Urbano de la Zona Metropolitana del Municipio de Acapulco de Juárez, Guerrero. ${ }^{2}$ Se formaron nueve subcomités especializados que analizaron a detalle los usos de suelo, vialidad, transporte, medio ambiente, protección civil, legislación urbana, etcétera. Dicho Plan, a diferencia de los anteriores, no iría solo, sino que estaría integrado a un Reglamento de Operación y Normas Complementarias. Posteriormente se agregó el Atlas de Peligros Naturales de la Ciudad de Acapulco, proporcionando así los que serían los primeros pasos para la consolidación de un sistema de protección civil y del proceso de gestión de riesgos en Acapulco. A pesar de este importante esfuerzo, algunos puntos específicos, como la Zona Diamante, no se planearon con el mismo cuidado. El Plan

${ }^{2}$ Este Plan se presentó en 1975, incorporando diversas actualizaciones en 1987, 1993 y 1998 (PDUZMa, 2001). 
Parcial de Zona Diamante se empezó a impulsar en el año 2000, pero en 2007 todavía no había sido concluido, faltaba un ordenamiento ecológico territorial. Esto nos muestra las deficiencias con las que se ha hecho el desarrollo inmobiliario en nuestra zona de estudio, vacío que ha propiciado márgenes de acción para las desarrolladoras y para la burocracia responsable de su aplicación.

En los meses posteriores a la tormenta se generó una intensa discusión en distintos órganos de gobierno, el primero en pronunciarse fue el Congreso de la Unión, el 4 de septiembre de 2007, durante el primer periodo ordinario de la Cámara de Diputados, donde se presentaron tres puntos de coincidencia: 1) exigir a las empresas que indemnicen a los afectados por el desastre; 2) un exhorto al Ejecutivo para que realice una averiguación que deslinde responsabilidades en el otorgamiento de los permisos de construcción, y 3) que la Secretaría de Gobernación declare los espacios afectados como zona de desastre. Aparentemente estos acuerdos fueron extendidos a las autoridades para su seguimiento. Por su parte, el Congreso local también propuso una investigación para determinar la responsabilidad en la asignación de licencias de construcción (El Sur, 13 de noviembre de 2007).

Presionados por estos acuerdos, en periódicos del mes de noviembre se encuentran declaraciones de funcionarios de distintas instancias federales, estatales y municipales que tratan de evadir las responsabilidades; entre los argumentos que presentaron algunos funcionarios era que habían autorizado lo que a ellos correspondía, ya que las solicitudes estaban acompañadas por documentación aprobatoria extendidas por otras oficinas de gobierno (declaraciones del secretario de Medio Ambiente del estado, Sabas de la Rosa Camacho, El Sur, 27 de noviembre de 2007). El Congreso envió un exhorto a la Secretaría de Medio Ambiente y al gobierno de Acapulco a no extender y suspender licencias hasta que se tenga un ordenamiento ecológico territorial de la zona (El Sur, 6 de noviembre de 2007).

Se ventiló el caso del fraccionamiento La Marquesita, donde en marzo de 2007 la Secretaría de Medio Ambiente y Recursos Naturales (Semaren) autorizó en forma condicionada la construcción de 143 viviendas a la empresa Construcciones y Promociones S.A., con sede en Querétaro. En el expediente de la Comisión de Agua Potable y Alcantarillado del Municipio de Acapulco (CAPAMA) se advierte que en la zona no existe infraestructura de drenaje, por lo que la empresa tendría que gestionar ante la Comisión Nacional del Agua (CNA) la construcción de una planta tratadora (El Sur, 25 de noviembre de 2007). La discusión continuó en los días siguientes, caldeando el ambiente político y de incertidumbre. Indudablemente éste fue el marco de los procesos de reconstrucción en Llano Largo, que imprimía su sello también en las relaciones entre los 
diversos actores. A falta de un ordenamiento ecológico territorial, se recurrió a estudios hidrológicos realizados por técnicos de la Universidad Nacional Autónoma de México (UNAM), que los llevó a plantear la realidad de que el lugar no es propicio para la construcción de asentamientos, no sólo por el riesgo sino también por las afectaciones ambientales.

Debido a que entre el 28 de octubre y el 15 de diciembre del mismo año ocurrieron inundaciones de graves consecuencias en Tabasco y Chiapas, la atención y los recursos federales se desplazaron hacia estos lugares que habían tenido pérdidas totales, por ello el Plan DN III para Acapulco no fue declarado, los fondos para la reconstrucción nunca llegaron a los afectados.

Sin embargo sí se canalizó ayuda hacia las zonas afectadas por la tormenta tropical Henriette, pero para poder rehabilitar los dańos ocasionados por dicha tormenta, fue necesario que se reuniera el Comité de Evaluación de Daños de ésta, ${ }^{3}$ integrado por funcionarios de los tres niveles de gobierno, debido a que tanto el municipio como el estado se declararon incapaces de solventar los gastos que ocasionarían las obras de reparación. Aparentemente lo que guió las decisiones fue rehabilitar lo dañado por la tormenta, ya que al momento de decidir, sólo se inclinaron por el boulevard de Las Naciones, en la medida que el boulevard Cayaco-Puerto Marqués, dijeron, ya estaba dañado desde antes (arquitecto Guillermo Torres). Esta decisión ocasiono malestar entre la población que radica en el sector popular del corredor Cayaco-Puerto Marqués, así como de los habitantes de Llano Largo, quienes manifestaron que esto era un trato discriminatorio por parte del Estado, debido a sus condiciones económicas.

El vocero de la Secretaría de Desarrollo Urbano y Obras Públicas del Ayuntamiento de Acapulco, Manuel Malváez Rosillo, declaró que se invertirían 460 millones de pesos en este sector afectado de la Zona Diamante, para la elevación de los puentes que conectan el boulevard de Las Naciones con la carretera Cayaco-Puerto Marqués y con la Unidad Colosio, así como la elevación del borde que colinda con la Laguna Negra. También se invertirá en el desazolve de $20 \mathrm{~km}$ del río La Sabana, así como la protección de sus bordes laterales y la construcción de un colector pluvial de norte a sur, desde El Cayaco hasta el canal meándrico, es decir, el límite este del polígono objeto de este estudio (El Sur, 13 de diciembre de 2007). También se invirtió en la introducción de drenaje pluvial en el boulevard de Las Naciones durante 2008 (informe del Gobierno del Estado, 2008).

\footnotetext{
${ }^{3}$ Compuesto por Jorge Alejandro Corti Velázquez, del Fonden; Regino Sábila Flores de la Secretaría de Hacienda; Hortensia González Miranda, de Salud, y Patricia Adriana Hernández Miranda de la Sedesol, todos ellos funcionarios federales; y por parte de la Secretaría de Desarrollo Urbano y Obras Públicas del estado se presento Guillermo Torres Madrid y su par municipal Manuel Malváez Rosillo.
} 
Por su parte, la Sedesol del estado, recordándonos que el evento afectó a un radio mayor que Zona Diamante, aplicó 2'577,000 pesos para el Programa de Empleo Temporal en los municipios de Acapulco, Coyuca de Benítez y San Marcos, con lo que se apoyó a los afectados por la tormenta tropical Henriette. Se dijo que en Acapulco se pagaron 5,812 jornales y se atendió a 79 colonias con acciones de desazolve, limpieza de calles con maquinaria, descacharrización, fumigaciones para prevenir el dengue, así como la demolición de dos rocas, en las que participaron los habitantes de colonias y comunidades que fueron dañadas por las inundaciones (boletín de comunicación social, núm. 1109-07, 9 de noviembre del 2007). En nuestra zona de estudio la atención de la reconstrucción incluyó el apoyo con materiales de construcción y casas para los antiguos pobladores, esto último se hizo en colaboración con el Programa Hábitat.

A partir de estos datos se puede observar que los rubros de inversión pública fueron puestos en la rehabilitación del boulevard de Las Naciones y en la mitigación de daños menores en el río de La Sabana. El otro gran rubro fue la atención focalizada de la población pobre, en donde Llano Largo era un asentamiento más entre poblaciones rurales y urbanas, muchas de ellas colonias irregulares. Para la reconstrucción el Estado se enfoca en la rehabilitación económica y allí encamina sus esfuerzos. En cuanto a la mitigación y prevención de desastres entre la población pobre, se dedica también a la rehabilitación de infraestructura, a dar empleo y asistencia sanitaria, pero no se piensa en los espacios o condiciones sociales de riesgo. A este sector de la población se les atiende como receptores de los daños colaterales del desarrollo, como bien señala Lavell (2000).

También es importante analizar la política de desastres del estado. En el año 2006 se conformó la Secretaría de Protección Civil, a la que se dotó de la infraestructura para su funcionamiento. En el ámbito municipal, Acapulco cuenta también con una oficina de Protección Civil cuya labor se articula con dicha Secretaría en esta área; se considera que su trabajo es coordinar los aportes de otras secretarías y de la sociedad civil mediante la formación de brigadas de trabajo que actúan durante el desastre. Es un trabajo especializado que se concentra en el monitoreo y alerta temprana, así como en la atención de la emergencia, en un sentido fisicalista (Hewitt, 1983), lo que lleva a desestimar la atención de la vulnerabilidad generada por los procesos de desarrollo (Lavell, 1996).

El planteamiento organizativo incluye representantes de distintos organismos públicos y privados que participan en un evento extraordinario; es limitado en tanto las pautas por las que se rigen están encuadradas en el esquema político-administrativo sectorializado del estado, de tal suerte que no existe un trabajo de base con los comités locales de protección civil. Esto resultó evidente durante las inundaciones estudiadas, 
donde la organización espontánea de la población contribuyó al apoyo a los afectados y sólo se conformaron las brigadas de protección civil con presencia de locales hasta la llegada de este organismo a la zona de desastre, pero una vez terminado el trabajo esperado de la población, ésta fue enviada a los albergues.

La participación de Protección Civil estatal y municipal termina con la superación de la emergencia, y la fase de reconstrucción queda a cargo de otras secretarías de Estado, que son quienes gestionan y ejercen la inversión pública, aparentemente ya sin la idea articulada de prevención o mitigación de desastres. En este sentido, ni la sectorialización ni el esquema participativo limitado de la población contribuye a conformar una visión integral de la actividad de protección civil.

\section{Conclusiones}

La experiencia estudiada nos muestra algunos de los problemas de sustentabilidad que afectan el proyecto de desarrollo de Zona Diamante, pues a siete años de haberse iniciado, el plan carece de un ordenamiento ecológico territorial. Esto explica los problemas ambientales y de riesgo generados en el espacio estudiado, así como la anarquía presente en el otorgamiento de los permisos de construcción y en los procesos de construcción realizados por las empresas. La tarea de planificar y construir el territorio ha estado en manos del Estado y los grupos de poder económico, mientras que los sectores afectados por los cambios territoriales han permanecido como observadores y receptores de los daños provocados en su vida por esas decisiones no consultadas. El problema se vuelve mayor si observamos que el Estado además carece de instrumentos de control y seguimiento del cumplimiento de la normatividad urbana y ambiental, lo que agrega mayor vulnerabilidad a los escenarios de riesgo, en este caso ocasionados por las constructoras. En tanto nunca se llevó a cabo la búsqueda de responsables, ni se han cambiado los procedimientos ni se han impuesto controles una vez que se han extendido los permisos, las empresas siguen construyendo, lo que promete mayores desastres por inundación en el sector.

El enfoque centrado en la emergencia que caracteriza las políticas de atención de los desastres en Guerrero, lleva a procesos de reconstrucción que, como en el caso de Llano Largo, termina acentuando las diferencias. Por un lado la inversión pública se centra en la rehabilitación económica; los nuevos pobladores, por su parte, pueden reconstruir sus espacios habitacionales recurriendo a las aseguradoras; mientras que los sectores pobres tienen que conformarse con la ayuda focalizada que además no llega a todos. En este sentido, la política focalizada tampoco puede resar- 
cir a los antiguos pobladores de esos daños o efectos indeseables del desarrollo. Esta ayuda, además, se ve mermada por los problemas de corrupción o políticas clientelares que se presentan desde los organismos públicos. El resultado es más polarización y segmentación que sólo puede romperse con procesos organizativos y autogestionarios para enfrentar el riesgo desde las organizaciones locales.

El caso estudiado nos mostró que la heterogeneidad de los actuales pobladores no fue obstáculo para que se desarrollaran procesos organizativos, donde destacan los nuevos pobladores, quienes con mayor información, así como experiencia en organización y participación ciudadana han conseguido resignificar sus grupos de condóminos en instancias de gestión del riesgo, si bien limitadas en la medida que responden a los propios intereses. Sin embargo, la constante amenaza de inundaciones estacionales al parecer se vuelve un elemento potenciador de la organización que eventualmente puede cobijar a otros grupos de afectados.

La política de atención de los desastres es limitada, en tanto carece de un planteamiento organizativo incluyente, esto impide el desarrollo de procesos favorables a la construcción de liderazgos y procesos de ejercicio democrático de la ciudadanía que contribuyan a establecer una cultura de protección civil desde la base, donde se puedan generar reflexiones y percepciones de riesgo que a partir de la historia y su relación con el medio social y físico produzcan respuestas a las amenazas y a la vulnerabilidad existentes desde lo local.

\section{Bibliografía}

Beck, Ulrich (2006), La sociedad del riesgo. Hacia una nueva modernidad, Paidós, Barcelona.

Evans, Victoria (1994), "Percepción del riesgo y noción del tiempo", Desastres y Sociedad, 3, año 2, Red de Estudios Sociales en Prevención de Desastres en América Latina, ciudad de Panamá, pp. 3-13, <http://www.desenredado.org>, 5 de agosto de 2010.

García-Acosta, Virginia (2005), "El riesgo como construcción social y la construcción social del riesgo", Desacatos, 19, Centro de Investigaciones y Estudios Superiores en Antropología Social, México, pp. 11-24.

Hewitt, Kenneth (1983), "The idea of Calamity in a Tecnocratic Age", en Kenneth Hewitt (ed.), Interpretations of Calamity, Allen and Unwin, Londres, pp. 3-32. 
Jacinto, Estela, Dulce Quintero, Rocío López y América Rodríguez (2009), "La expropiación como utilidad pública: historia de un proceso en la franja costera de Acapulco", en América Rodríguez Herrera et al. (coords.), Los retos del desarrollo en Acapulco, Unidad de Desarrollo Regional-Universidad Autónoma de Guerrero, Chilpancingo, pp. 39-56.

Juárez-López, Ana Laura (2009), "Manejo de residuos sólidos urbanos para los cauces fluviales de la zona urbana y suburbana de Acapulco, Guerrero", tesis de doctorado, Universidad Autónoma de Guerrero, Chilpancingo.

Lavell, Allan y Eduardo Franco (1996), Estado, sociedad y gestión de los desastres en América Latina: en busca del paradigma perdido, La Red-flacso, Lima.

Lavell, Allan (2000), "Desastres durante una década: lecciones y avances conceptuales y prácticos en América Latina (1990-1999)", Anuario Político y Social de América Latina, 3, La Red-flacso, pp. 1-32.

Lavell, Allan (2002), "Desastres urbanos: una visión global", en Mario Lungo, Riesgos urbanos, Istmo, San Salvador, pp. 17-28.

Mairal-Buil, Gaspar (1999), "Los conflictos del agua y la construcción del riesgo", en Pedro Arrojo Agudo y Francisco Javier Martínez Gil (eds.), El agua a debate desde la universidad: hacia una nueva cultura del agua, I Congreso Ibérico sobre Gestión y Planificación de Aguas, Zaragoza, pp. 605-616.

Maskrey, Andrew (1983), Los desastres no son naturales, Tercer Mundo-La Red, Bogotá.

pDuzma (2001), Plan de Desarrollo Urbano de la Zona Metropolitana de Acapulco de Juárez, H. Ayuntamiento Constitucional, Acapulco.

Ríos, Diego y Ana María Múrgida (2004), "Vulnerabilidad cultural y escenarios de riesgo por inundación", Espaço e Tempo, 16, Grousp, São Paulo, pp. 181-192.

Rodríguez-Herrera, América, Dulce María Quintero Romero y Rocío López Velasco (2010), "Inventario de fuentes de contaminación en Llano Largo, en la cuenca del río La Sabana, Acapulco", po- 
nencia presentada en el XV Congreso de Amecider, Tabasco, 16-19 de noviembre.

Rodríguez-Velásquez, Daniel (2003), “De la teoría a la práctica, sociedad civil y desastres", ponencia presentada en el Tercer Congreso Internacional Red Nacional de Investigación Urbana, Balance y Perspectivas del Análisis Territorial, Puebla, 22-23 de septiembre.

Ruiz-Massieu, José Francisco (1989), Acapulco diamante. El Acapulco del siglo XXI, Gobierno del Estado de Guerrero, Chilpancingo.

Wilches-Chaux, Gustavo (1983), "Pensar globalmente, la vulnerabilidad global. Bogotá”, <http://osso.univalle.edu-co>, 5 de agosto de 2010.

Recibido: 28 de enero de 2009.

Reenviado: 21 de octubre de 2010. Aceptado: 1 de febrero de 2011.

América Rodríguez-Herrera. Es doctora en antropología social por la Universidad Iberoamericana; licenciada en antropología por la Escuela Nacional de Antropología e Historia de la ciudad de México, y maestra en sociología por la Universidad de Costa Rica. Actualmente es miembro del Sistema Nacional de Investigadores (sNI), nivel I. Se desempeña como docente-investigadora en la Unidad de Desarrollo Regional de la Universidad Autónoma de Guerrero. Sus líneas de investigación son: desarrollo urbano y cambios en el territorio, participación ciudadana, manejo de recursos naturales y sustentabilidad. Entre sus publicaciones destacan: "Exclusión, identidad y acceso al agua", en R. Boelens, A. Guevara, J. Hendriks y J. Hoogesteter, Pluralismo legal, reforma hidrica y politicas de reconocimiento, vol. 13, Universidad de Wageningen-CEPAL, pp. 145-264 (2007); "Organizaciones sociales: nuevos actores políticos en Guerrero", Revista Política y Cultura, 30, uAM, Xochimilco, México, pp. 33-36 (2008); "Agua e identidad étnica en Izalco, República de El Salvador", Agricultura Sociedad y Desarrollo, 4 (2), Colegio de Postgraduados, Campus Texcoco, México, pp. 83-103 (2007).

Manuel Ignacio Ruz-Vargas. Es profesor-investigador de tiempo completo, con perfil deseable Promep, en la Unidad Académica de Arquitectura y Urbanismo (UAAU) de la Universidad Autónoma de Guerrero (Uagro); es profesor invitado en el Centro de Investigación y Posgrado en Estudios Socioterritoriales (CIPES), donde imparte el curso de urbani- 
zación y ciudades en la maestría en estudios socioterritoriales de la Uagro. Es licenciado en arquitectura por el Instituto Tecnológico de Acapulco (ITA); maestro en ciencias sociales por la Uagro; actualmente cursa el doctorado en desarrollo regional en la Uagro. Es miembro de la Comisión Técnica del Área Humanística Social del Consejo de Ciencia y Tecnología del Estado de Guerrero (Cecyteg); forma parte del Padrón Estatal de Investigadores del Estado de Guerrero y de la Mesa Directiva de la Sociedad Mexicana de Ingeniería Sísmica (smis), Delegación Guerrero; es coordinador del Programa Institucional de Tutorías en la UAAU y miembro de la Comisión de Asuntos Académicos en el Consejo Universitario de la Uagro. Sus líneas de investigación actuales son: planeación y desarrollo urbano sustentable, y percepción del riesgo ante los desastres. Entre sus publicaciones destacan: "Vulnerabilidad social: la Caja Negra de Paulina", tesis de maestría, Universidad Autónoma de Guerrero, Chilpancingo (2005); en coautoría, "Hechos y deshechos en la Cuenca del Río La Sabana”, en Dinámica de la gestión del agua en el Consejo de Cuenca de la Costa de Guerrero, Comisión Nacional del Agua-Universidad Autónoma de Guerrero, ciudad, pp. 154-172 (2009); en coautoría, "Desarrollo urbano, planeación y construcción de riesgos”, en América Rodríguez Herrera (coord.), Las inundaciones en Llano Largo, Acapulco: riesgo, turismo y desarrollo, Plaza y Valdez-Conacyt-Unidad de Ciencias de Desarrollo Regional-Universidad Autónoma de Guerrero, México, pp. 51-92 (2011); "El último bastión del Mar del Sur", Revista de Arquitectura, Diseño y Urbanismo, 9, Universidad Autónoma de Morelos-Universidad Autónoma de Guerrero, Cuernavaca, Morelos, pp. 112-125 (2011); en coautoría, "San Diego, un fuerte con valor excepcional universal", Revista Tlamati, 3, Universidad Autónoma de Guerrero, México, pp. 50-55 (2011).

Berenise Hernández-Rodríguez. Es maestra en desarrollo regional por la Universidad Autónoma de Guerrero; licenciada en sociología de la educación y la comunicación por la Universidad Autónoma de Guerrero, actualmente estudia doctorado en desarrollo rural en la Universidad Autónoma del Estado de Morelos. Entre sus publicaciones destacan: en coautoría: "Sistemas de riego en La Cañada de Haumuxtitlán: tradición y actualidad", Tecnología y Ciencias del Agua, i (4), México, pp. 75-88 (2010); en coautoría, "La tormenta tropical Henrriette y las inundaciones en Costa Dorada en el ejido de Llano Largo ¿Desastre? La respuesta de la gente", en Amecider, Memorias del $13^{\circ}$ Encuentro nacional sobre el desarrollo regional en México: problemática regional en México: hacia una agenda para el desarrollo, Asociación Mexicana para el Desarrollo Regional, A.C., México. 